\title{
Bayesian Adaptive Exploration
}

\author{
Thomas J. Loredo
}

Dept. of Astronomy, Cornell University, Ithaca, NY 14853, USA

\begin{abstract}
I describe a framework for adaptive scientific exploration based on iterating an Observation-Inference-Design cycle that allows adjustment of hypotheses and observing protocols in response to the results of observation on-the-fly, as data are gathered. The framework uses a unified Bayesian methodology for the inference and design stages: Bayesian inference to quantify what we have learned from the available data and predict future data, and Bayesian decision theory to identify which new observations would teach us the most. When the goal of the experiment is simply to make inferences, the framework identifies a computationally efficient iterative "maximum entropy sampling" strategy as the optimal strategy in settings where the noise statistics are independent of signal properties. Results of applying the method to two "toy" problems with simulated data - measuring the orbit of an extrasolar planet, and locating a hidden one-dimensional object-show the approach can significantly improve observational efficiency in settings that have well-defined nonlinear models. I conclude with a list of open issues that must be addressed to make Bayesian adaptive exploration a practical and reliable tool for optimizing scientific exploration.
\end{abstract}

\section{INTRODUCTION}

The classical paradigm for the scientific method follows a rigid sequence of hypothesis formation, followed by experiment and then analysis. It bears little resemblance to the adaptive, self-adjusting behavior of the human brain, which learns from experience incrementally, making decisions and adjusting questions on-the-fly. The classical paradigm has served science well, but there are many circumstances where what has been learned from past data could be profitably used to alter the collection of future data to more efficiently address the questions of interest. In this paper I describe an approach for developing such adaptive observing strategies building on existing ideas from the theory of Bayesian experimental design.

The idea that use of partial knowledge can improve the design of experiments has long been recognized in statistics; there are well-developed theories of experimental design using both the frequentist and Bayesian approaches to statistics (good entry points to the large literature are [1, 2, 3] for frequentist design, and [4, 5] for Bayesian design). Unfortunately, practice has lagged theory, largely due to the complicated calculations required for rigorous experimental design, particularly in adaptive settings where many designs must be calculated. Until recently most work focused on classes of problems that are analytically tractable (e.g., linear models with normal errors, and, in Bayesian design, with flat or conjugate priors). Treatment of nonlinear models was typically handled only approximately, by linearizing about a best-fit model. These limitations have discouraged application to problems of interest to astronomers and physicists, which often have substantial nonlinearities and other complications. In addition, the gains offered by optimal designs in analytically tractable settings are often only modest. 
Finally, in these settings frequentist and Bayesian designs are the same or very similar, suggesting (erroneously) that the two approaches have little distinguishing themselves from each other.

In recent years computational and theoretical developments finally enable one to undertake rigorous nonlinear Bayesian design in complicated settings. Most important for the approach described here are:

- The discovery that a wide and interesting class of design problems can be analytically simplified, revealing that the optimal observing strategy obeys a relatively simple maximum entropy sampling criterion [6, 7];

- The development of flexible and rigorous methods for Bayesian computation based on sampling posterior distributions for models and parameters with Markov chain Monte Carlo (MCMC) algorithms, allowing rigorous optimal design with nonlinear models [8, 9, 10, 11].

In the following section I describe the basic principles behind Bayesian design in an adaptive setting - here dubbed Bayesian adaptive exploration (BAE) — and highlight how recent developments open the door to applications of realistic complexity. The subsequent two sections describe results of two proof-of-concept calculations showing that BAE could improve observational efficiency in a variety of problems in the physical sciences and engineering. The first example concerns measuring the orbit of an extrasolar planet, and is motivated by the needs of the upcoming Space Interferometry Mission (SIM) which will astrometrically survey nearby stars for evidence of planets, including a search for Earth-like planets around Sun-like stars (see [12] for further discussion of the possible role of BAE for SIM). The second example concerns locating a hidden one-dimensional object, and is motivated by the need to optimally deploy a variety of sensing technologies to efficiently and accurately locate and identify buried landmines. The final section briefly describes the main open issues that must be addressed to make BAE a truly useful tool for optimal scientific exploration.

\section{BAYESIAN ADAPTIVE EXPLORATION}

BAE iterates an Observation-Inference-Design cycle depicted in Figure 1. In the observation stage, new data are obtained based on an observing strategy produced by the previous cycle of exploration. The inference stage synthesizes the information provided by previous and new observations to assess hypotheses of interest. This synthesis produces interim results such as signal detections, parameter estimates, or object classifications. Finally, in the design stage the results of inference are used to predict future data for a variety of possible observing strategies; the strategy that offers the greatest predicted improvement in inferences (subject to any resource constraints) is passed on to the next Observation-Inference-Design cycle. The observation stage will follow the observation procedure dictated by the nature of the experiment and need not be discussed further here. In the remainder of this section I outline the components of the inference and design stages, the stages where the tools of Bayesian statistics enter the process. 


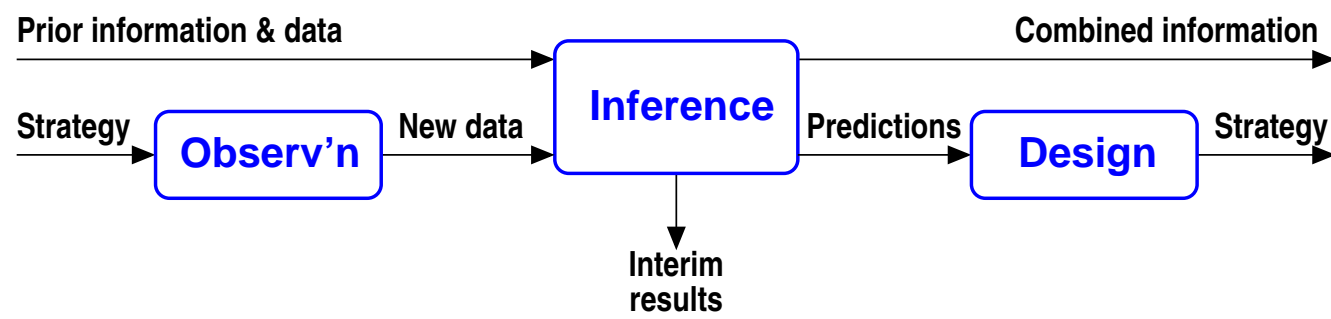

FIGURE 1. Information flow through one cycle of the adaptive exploration process.

\section{Inference Stage}

BAE uses the tools of Bayesian inference for the inference stage. Readers of this volume are most likely familiar with these tools. We review them briefly here in order to establish notation. ${ }^{1}$

We address inference questions by calculating probabilities for the hypotheses of interest $\left(H_{i}\right)$ given the available data $(D)$ and the underlying modelling assumptions $(M)$; the resulting posterior probability distribution is denoted $p\left(H_{i} \mid D, M\right)$. Bayes's theorem expresses it in terms of more directly calculable probabilities as follows;

$$
p\left(H_{i} \mid D, M\right)=p\left(H_{i} \mid M\right) \frac{p\left(D \mid H_{i}, M\right)}{p(D \mid M)},
$$

where $p\left(H_{i} \mid M\right)$ is the prior probability distribution for $H_{i}, p\left(D \mid H_{i}, M\right)$ is the sampling distribution for the data, and $p(D \mid M)$ is the prior predictive distribution for the data. In inference one is interested in how the posterior varies with $H_{i}$, with the data fixed at the observed values. Thus what is of most interest about the sampling distribution is how it varies with respect to the $H_{i}$, not the data. Considered as a function of $H_{i}$, it is called the likelihood for $H_{i}$, and denoted $\mathscr{L}\left(H_{i}\right) \equiv p\left(D \mid H_{i}, M\right)$. The prior predictive distribution has no dependence on $H_{i}$ and thus plays the role of a normalization constant. It can be calculated by summing the numerator in Bayes's theorem:

$$
p(D \mid M)=\sum_{i} p\left(H_{i} \mid M\right) \mathscr{L}\left(H_{i}\right)
$$

This is just the average likelihood for $H_{i}$, with the prior giving the averaging weight. Accordingly, $p(D \mid M)$ is sometimes called the average, global, or marginal likelihood.

When the hypotheses are labeled by one or more continuous parameters, $\theta$, the probability for any hypothesis about $\theta$ can be calculated from the posterior probability density, $p(\theta \mid D, E)$, usually via an integral of some sort. Bayes's theorem holds for posterior densities as well as for discrete distributions; the posterior density is proportional to the product of the prior density $p(\theta \mid M)$ and the likelihood function $\mathscr{L}(\theta)$. The average likelihood is given by an equation like equation (2), but with the sum replaced by an integral.

${ }^{1}$ For introductions to Bayesian inference, see [13, 14, 15], and our Bayesian Inference in the Physical Sciences web site (http://www. astro.cornell.edu/staff/loredo/bayes/). 
Several aspects of the Bayesian approach to statistical inference make it particularly suitable for adaptive design; two are especially worth highlighting. First is the presence of prior probabilities. Prior probabilities allow one to take into account information available before embarking on an experiment. But more importantly for our purposes, they allow for straightforward modelling of incremental, adaptive learning: the posterior probability from current observations becomes the prior probability for the next stage of exploration. In this way uncertainty can be rigorously carried through the entire exploration process, summarizing the totality of available information in a flexible, updatable way that can guide further exploration.

A second element of Bayesian inference particularly useful for experimental design is the ability to marginalize, that is, to eliminate dimensions of a problem that are not of immediate interest while fully accounting for the effects of their uncertainty on the quantities that are of interest. The most common use of marginalization arises when modelling the data requires introduction of parameters that are not directly of scientific interest (e.g., background rates, uncertain detector efficiencies). Separate $\theta$ into the interesting parameters, $\psi$, and the uninteresting nuisance parameters, $\phi$. The inference we report for $\psi$ is the marginal distribution, found by integrating the full posterior distribution over the nuisance parameters,

$$
p(\psi \mid D, M)=\int d \phi p(\psi, \phi \mid D, M) .
$$

Such calculations are useful in the inference stage because nuisance parameters are present in nearly all real inference problems. As we will see below, marginalization also proves to be crucial for design because it allows accurate prediction of future data. Having so straightforward a way to eliminate nuisance parameters while accounting for the effects of their uncertainty on inferences is an important advantage of the Bayesian approach. However, it comes with a cost: the integrals required are often challenging, particularly when the parameter space has more than a few dimensions.

All the probabilities above are conditional on the modelling assumptions or background information, $M$. It must include all information needed to evaluate the prior and likelihood, including such things as specification of the hypothesis space, the sample space (space of possible data sets), and the model or models connecting these (e.g., a parameterized theoretical model for the phenomenon of interest and distributional assumptions about noise). It could also include information from other experiments. It is important to note that $M$ must also include specification of basic properties of the experiment (e.g., size of the data sample, locations of samples, etc.). In the design stage, we will be concerned with how the posterior changes in part as a function of some of these elements of $M$.

\section{Design Stage}

The goal of the design stage is to specify a new experiment (e.g., the location in time or space for a next sample or set of samples) that will provide new data that will best serve our scientific aims. Of course, we do not know what new data will actually be obtained, 
so our choice must be based on predictions of what various candidate experiments might see. In a Bayesian framework the key ingredient for this is the predictive distribution for the future data; Bayesian inference provides tools for calculating this. Once we can make predictions about results of experiments, it only remains to choose which experiment is best. This is not an inferential task, but rather a decision problem, and the proper tool for it is Bayesian decision theory [13, 15]. We now describe the prediction and decision aspects of experimental design in turn.

A candidate experiment will be described by one or more parameters, specifying things like sample location in space or time. Denote these parameters by $e$. If we knew the parameters, $\theta$, describing the phenomenon of interest, it would be easy to make predictions about the data, $d$, we expect from experiment $e$; we simply evaluate the sampling distribution for that data, $p\left(d \mid \theta, M_{e}\right)$, where the subscript on the background information makes explicit that this information includes specification of the sample location. Of course, we are doing the experiment because we do not know $\theta$. But information about $\theta$ is available from the inference stage in the form of the posterior based on earlier data, $p(\theta \mid D, M)$. A predictive distribution for $d$ that uses this partial knowledge about $\theta$ would condition on the known data, $D$, rather than on the unknown $\theta$; we seek $p\left(d \mid D, M_{e}\right)$. This predictive distribution can be evaluated by introducing the unknown $\theta$, and marginalizing;

$$
\begin{aligned}
p(d \mid D, M) & =\int d \theta p\left(d, \theta \mid D, M_{e}\right) \\
& =\int d \theta p\left(d \mid \theta, M_{e}\right) p\left(\theta \mid D, M_{e}\right),
\end{aligned}
$$

where for the second line we used the product rule, recognizing that $p\left(d \mid \theta, D, M_{e}\right)=$ $p\left(d \mid \theta, M_{e}\right)$, that is, once $\theta$ is given, conditioning on $D$ is irrelevant. The integrand in equation (4) is the product of two readily available quantities, the sampling distribution for new data, and the posterior distribution produced by the previous inference stage.

Now we must use properties of the predictive distribution to decide which experiment-which value of $e$-is best. This requires more than just the tools of inference. In reaching a decision, it is not enough to consider only the uncertainties of possible outcomes; consequences must also be taken into account. One might be willing to bet on an improbable outcome if the payoff is large if it occurs and the loss is small if it does not. Consequences are quantified via the utility of a decision. The utility depends both on the possible actions we are deciding between (to bet or not bet in the example just given) and on the possible outcomes (determining whether the bet is lost or won). Denoting actions by $a$ and outcomes by $o$, the utility function defining a decision problem can be written as a function of both, $U(o, a)$, and our task is to decide on $a$ amidst uncertainty about $o$.

To make the best decision, decision theory specifies that one first calculate the $e x$ pected utility of the possible decisions, averaging the utility using the probabilities of the possible outcomes:

$$
E U(a)=\sum_{o} p\left(o \mid I_{a}\right) U(a, o),
$$

where $I_{a}$ denotes whatever information is available about the outcomes, with the $a$ subscript making explicit the possibility that the choice of action affects the probability 
of the outcomes. The best action, $\hat{a}$, is the one that maximizes the expected utility,

$$
\hat{a}=\arg \max _{a} E U(a)
$$

In experimental design, the possible actions are the possible experiments we might perform, indexed by $e$, and the possible outcomes are the values of future data from that experiment, $d$. An experimental design problem thus requires specification of a utility, $U(d, e)$, that balances the value of $d$ for achieving the scientific goals against possible costs of various experiments. Once the utility is specified, the best experiment is the one that maximizes

$$
E U(e)=\int d d p\left(d \mid I_{e}\right) U(d, e)
$$

The conditioning information most typically includes a parameterized model for the phenomenon producing the data, and possibly values of already collected data; so the probability we need is the predictive distribution, $p\left(d \mid D, M_{e}\right)$. Using equation (4), we can write the expected utility as,

$$
E U(e)=\int d \theta p\left(\theta \mid D, M_{e}\right) \int d d p\left(d \mid \theta, M_{e}\right) U(d, e) .
$$

To proceed we must specify $U(d, e)$. In disciplines such as econometrics or biometrics, there are obvious costs and benefits of decisions; consequently, decision theory is more prominent in these disciplines than in the physical sciences, where the goal of a study is usually not to reach a formal decision, but to report the implications of the data for various hypotheses. That is, in the physical sciences we are primarily concerned with inference, with reporting the information conveyed by the entire posterior distribution, not with achieving a specific goal with an assigned value. But we can still use decision theory to determine the best experiment to perform if we can come up with a utility that measures how well the experiment improves our inferences.

In 1956, Lindley described how one could use tools from information theory in a Bayesian framework to compare experimental designs when one's purpose is simply to gain knowledge about a phenomenon [16]. He later incorporated these ideas into the more general theory of Bayesian experimental design outlined above, first described in his influential 1972 review of Bayesian statistics [17]. Although non-Bayesian methods for optimal design predate Lindley's work (standard references are [1, 2, 3]), the Bayesian approach provides a more fundamental rationale for many earlier methods, and unifies and generalizes them (see [4] for discussion of the relationships between Bayesian and non-Bayesian design).

Lindley suggested that if the goal is to learn about $\theta$, a natural utility function is the information in the final posterior distribution for $\theta$, as measured by information theory,

$$
U(d, e)=\int d \theta p\left(\theta \mid d, D, M_{e}\right) \log p\left(\theta \mid d, D, M_{e}\right),
$$


where $p\left(\theta \mid d, D, M_{e}\right)$ is the posterior for $\theta$ including future data $d$, and the right hand side is just the negative Shannon entropy of this distribution. ${ }^{2}$ Using equation (8), the expected information from experiment $e$ is,

$$
\begin{aligned}
E U(e)= & \int d \theta p\left(\theta \mid D, M_{e}\right) \int d d p\left(d \mid \theta, M_{e}\right) \\
& \times \int d \theta^{\prime} p\left(\theta^{\prime} \mid d, D, M_{e}\right) \log p\left(\theta^{\prime} \mid d, D, M_{e}\right) .
\end{aligned}
$$

The best experiment is the one that maximizes the expected information. If there are definite, variable costs associated with various choices of $e$, they can be subtracted from the utility. Up to this possible generalization, we have completed specification of the design stage.

\section{Implementation}

In the three decades since Lindley advocated designing to maximize information, the theory of design has matured significantly. But as noted in Toman's recent review of Bayesian design, "unfortunately much of the work in this area remains purely theoretical" [5]. This is largely due to the computational complexity of Bayesian design, an obstacle noted already in Lindley's foundational work. In experimental design, one must account for both uncertainty regarding the hypotheses under consideration, and uncertainty about the values of future data-equation (8) has integrals over both $\theta$ and $d$. For the former, one must perform the difficult parameter space integrals that are characteristic of Bayesian inference [19]; for the latter, one must additionally integrate over the sample space as is typically done in frequentist calculations (e.g., by Monte Carlo simulation of data). In a sense, experimental design is the arena in which the Bayesian and frequentist outlooks meet, producing problems with the combined complexity of both approaches. In addition, when designing to maximize information, the utility itself requires a nontrivial parameter space integration (the integral over $\theta^{\prime}$ in equation (10), adding to the complexity.

The computational complexity of Bayesian experimental design has led researchers to focus on problems where all or most of the needed integrals are analytic, e.g., data with additive Gaussian noise and linear models. In particular, little work exists rigorously handling nonlinear models (most nonlinear design work relies on linearization about a best-fit model; e.g., [18, 20]). For linear models with additive Gaussian noise, it is known that design does not depend on the values of the available data, only on their noise levels and noise correlations [18] (this is because the data values affect the location but not the

\footnotetext{
${ }^{2}$ For a Gaussian distribution with standard deviation $\sigma$, the Shannon entropy is proportional to $-\log (\sigma)$ and thus increases with decreasing $\sigma$ as one would expect of a measure of information; but it is a more general measure of spread than the standard deviation. To be formally correct, the argument of the logarithm in equation (9) should be divided by a measure on the parameter space so the argument is dimensionless; this has no significant effect on our results. An alternative definition of information is the cross-entropy or Kullback-Leibler divergence between the posterior and prior; it gives the same results as the Shannon entropy for this calculation [18].
} 
width of the posterior for linear models, and Shannon information measures the width). This limits the improvements one can achieve using optimal designs with such models.

In recent years, two advances have set the stage for rigorous Bayesian design with nonlinear models. On the theoretical front, investigators have found a broad and interesting class of problems for which the expected information expression in equation (10) can be analytically simplified, reducing the dimensionality of the integrals needed. On the computational front, investigators have used the technique of posterior sampling, particularly via MCMC methods, to calculate and report quantities needed for nonlinear design without any approximation of the integrands. We review these developments in turn.

\section{Maximum Entropy Sampling}

Let us explore the structure of the expected information expression analytically. Information is a functional, a mapping from functions to real numbers. Our manipulations will involve the information in various distributions, so we need a notation that is both compact but clearly shows what distribution is being used for an information calculation. Let $\mathscr{I}[x \mid I]$ denote the information in the distribution $p(x \mid I)$, so that

$$
\mathscr{I}[x \mid I] \equiv \int d x p(x \mid I) \log p(x \mid I)
$$

With this notation, the expected utility, equation (7), can be written,

$$
E \mathscr{I}(e)=\int d d p\left(d \mid I_{e}\right) \mathscr{I}\left[\theta \mid d, I_{e}\right]
$$

The integrand of equation (12) includes the information in the posterior distribution. This can be rewritten in terms of the information in other distributions using Shannon's theorem, the information theory analog to Bayes's theorem. As with the proof of Bayes's theorem, we establish Shannon's theorem by looking at the joint distribution for $\theta$ and $d$, and using the product rule to factor it. Dropping the common conditioning proposition $I_{e}$ for the moment,

$$
\begin{aligned}
\mathscr{I}[d, \theta] & =\int d d \int d \theta p(d, \theta) \log p(d, \theta) \\
& =\int d d \int d \theta p(d, \theta) \log p(\theta)+\int d d \int d \theta p(d, \theta) \log p(d \mid \theta) \\
& =\mathscr{I}[\theta]+\int d \theta p(\theta) \mathscr{I}[d \mid \theta],
\end{aligned}
$$

where for the first term we used the fact that $d$ appears only in a $p(d \mid \theta)$ factor in the first factor of the second line; this integrates to unity. Repeating the calculation but switching the order of factoring $d$ and $\theta$ we similarly find,

$$
\mathscr{I}[d, \theta]=\mathscr{I}[d]+\int d d p(d) \mathscr{I}[\theta \mid d]
$$


Equating the right hand sides of equations (13) and (14) gives Shannon's theorem. But note that the last term in equation (14) is just the expected information, equation (12). Thus Shannon's theorem shows that

$$
E \mathscr{I}(e)=\mathscr{I}\left[\theta \mid I_{e}\right]+\int d \theta p\left(\theta \mid I_{e}\right) \mathscr{I}\left[d \mid \theta, I_{e}\right]-\mathscr{I}\left[d \mid I_{e}\right] .
$$

In words, the expected information from an experiment is the information in the prior, plus the average information in the sampling distribution, minus the information in the predictive distribution.

In most cases the information in the prior (i.e., the posterior from consideration of existing data) will not depend on the sampling scheme for future data, so the first term in equation (15) will be constant. More subtly, in many cases the information in the sampling distribution will also be independent of the sample location. For example, often the sampling distribution describes the influence of noise on the data, with noise properties that are independent of the level of whatever signal the noise is added to (this would be true of many experiments with additive noise, but not of experiments with Poisson statistics except in the limit where background dominates signal). In these cases, the second term is also constant. As a result, only the last term depends on $e$; writing it out, we have,

$$
E \mathscr{I}(e)=C-\int d d p\left(d \mid D, M_{e}\right) \log p\left(d \mid D, M_{e}\right) .
$$

The best experiment maximizes this expression. Noting the minus sign, this means that the best experiment is the one whose predictive distribution has maximum entropy. Sampling according to this criterion is thus called maximum entropy sampling; despite its simplicity this criterion was discovered in its full generality only recently by Sebastiani and Wynn [6, 7]. Entropy is large for distributions that are broad and uninformative. Thus this is an eminently reasonable criterion; it tells us that we will learn the most by sampling where we know the least.

Besides providing an intuitive understanding of what Bayesian design accomplishes, equation (16) significantly simplifies the needed computations. The predictive distribution appearing in equation (16) requires a parameter space integral, so calculating the $e$-dependent part of $E \mathscr{I}(e)$ requires nested sample space and parameter space integrals. But the full expected information expression, equation (10), involves a further nested parameter space integral. When the conditions for maximum entropy sampling apply, we are spared of one level of parameter space integration.

\section{Posterior Sampling}

Even when the design problem simplifies to a maximum entropy sampling problem, we must evaluate equation (16) as a function of $e$. In situations such as those described below where BAE is being implemented sample-by-sample, the $d$ integration will be of low dimension (perhaps just a single dimension, as in the examples below). But the predictive distribution appearing in equation (16) requires a parameter space integration 
that can be challenging to do with models that have more than a few dimensions. Since about 1990, enormous progress has been made in finding methods to perform such integrals numerically without having to approximate the integrands. The most influential methods use posterior sampling. One creates a psuedorandom number generator that produces parameter values sampled from the full posterior distribution. Once a set of such values is available, one can estimate many quantities of interest by simple manipulation of the samples. The samples themselves also provide a visually appealing display of the structure of the posterior. The most popular and most flexible class of methods for obtaining posterior samples are MCMC methods, though for problems of small or moderate dimension simpler methods can be feasible.

As an example of how posterior sampling can facilitate nonlinear design, imagine we have an algorithm that provides us with a set of $N$ samples, $\left\{\theta_{i}\right\}$, from the posterior for $\theta$. Then the value of the predictive probability density for a particular value of $d$ can be estimated as follows;

$$
\begin{aligned}
p\left(d \mid D, M_{e}\right) & =\int d \theta p\left(\theta \mid D, M_{e}\right) p\left(d \mid \theta, M_{e}\right) \\
& \approx \frac{1}{N} \sum_{i} p\left(d \mid \theta_{i}, M_{e}\right)
\end{aligned}
$$

that is, simply average the values of the sampling distribution for $d$ conditioned on the posterior samples of $\theta$. Call this estimate $\tilde{P}(d)$. In addition to calculating the value of the predictive density, the posterior samples allow us to sample a $d$ value from it. We simply iterate the following two steps to generate, say, $M$ samples, $\left\{d_{j}\right\}$ :

- Sample the posterior distribution, yielding a parameter point, $\theta_{i}$. (If a pool of posterior samples is already available, just sample uniformly from that pool.)

- Sample $d_{j}$ from the sampling distribution for $d$ conditioned on $\theta_{i}, p\left(d \mid \theta_{i}, M_{e}\right)$.

The last step is often trivial; e.g., for data modeled as a signal with additive noise with a normal distribution, one simply draws a sample from the normal distribution (suitably scaled and shifted).

With these two ingredients, we can estimate equation (16) in the familiar Monte Carlo way;

$$
E \mathscr{I}(e) \approx \frac{1}{M} \sum_{j} \log \tilde{P}\left(d_{j}\right),
$$

where we have dropped the uninteresting constant. This is the approach used for the calculations described below, evaluating $E \mathscr{I}(e)$ over a grid of $e$ values and locating the maximum directly. As noted in the final section, other investigators have developed other algorithms that inventively use posterior sampling, in some cases to "sample" over the $e$ dimension of the problem in a way that targets attention to the optimal design.

The combination of analytical simplification via maximum entropy sampling (where possible) and use of posterior sampling algorithms is finally making rigorous nonlinear design feasible. We now turn to some simplified but nontrivial examples to explore the potential of BAE to improve the effectiveness of exploration in an adaptive framework. 


\section{EXAMPLE: EXTRASOLAR PLANET MEASUREMENTS}

Consider the problem of optimally scheduling observations of a star in order to characterize the orbit of a planet detected via radial velocity measurements of the Keplerian reflex motion of the star. The data are modeled by

$$
d_{i}=V\left(t_{i} ; \tau, e, K\right)+n_{i}
$$

where $n_{i}$ is the noise contribution to datum $i$, and $V\left(t_{i} ; \tau, e, K\right)$ gives the Keplerian velocity along the line of site as a function of time $t_{i}$ and of the orbital parameters $\tau$ (period), $e$ (eccentricity), and $K$ (velocity amplitude); for simplicity three purely geometric parameters are suppressed. This function is strongly nonlinear in all variables except $K$. When the eccentricity vanishes, it is simply a sinusoid; for nonzero eccentricity it has a more complicated periodic shape. Our goal is to learn about the parameters $\tau, e$ and $K$.

Figure 2 shows results from one Observation-Inference-Design cycle, using simulated data. Figure 2 a depicts the initial observation stage. The points show the data from a "setup" observation; observations were made at 10 equispaced times, and the error bars indicate the noise standard deviation (the noise distribution is Gaussian with zero mean and $\left.\sigma=8 \mathrm{~m} \mathrm{~s}^{-1}\right)$. The curve shows the true orbit with typical exoplanet parameters $\left(\tau=800 \mathrm{~d}, e=0.5, K=50 \mathrm{~ms}^{-1}\right)$.

Figure $2 \mathrm{~b}$ shows some results from the inference stage using these data. Shown are 100 samples from the marginal posterior density for $\tau$ and $e$. In a more careful calculation, we would use more samples and smoothing to find contours of credible regions; here it suffices to note that the displayed cloud of points should conservatively bound a $90 \%$ credible region. The period and eccentricity are usefully constrained by the 10 data points, but there is significant uncertainty that would not be well described by a Gaussian distribution (even correlated). Figure $2 \mathrm{c}$ shows how easily a complicated marginal distribution can be found using the samples; it displays the marginal distribution for two interesting physical parameters of the system, the planet's semimajor axis, $a$, and $m \sin i$, the product of its mass and the sine of its orbital inclination. These are each nonlinear functions of the three model parameters. To produce Figure $2 \mathrm{c}$ we simply evaluated these functions for each of the 100 samples of $(\tau, e, K)$ already produced; this is much simpler than numerically evaluating the multiple integral defining the marginal distribution over a $(m \sin i, a)$ grid. By reporting the actual sample values, other investigators could use the results of these observations in their own calculations and fully account for the uncertainties simply by evaluating any quantities of interest over the set of samples.

Figure $2 \mathrm{~d}$ illustrates the design stage. The thin curves display the uncertainty in the predictive distribution as a function of sample time; they show the $V(t)$ curve associated with 15 of the parameter samples from the inference stage. The spread of these curves at a particular time displays the uncertainty in the predictive distribution at that time. The Monte Carlo calculation of the actual expected information using all 100 samples is plotted as the thick curve (right axis, in bits, offset so the minimum is at 0 bits). The curve peaks at $t=1925 \mathrm{~d}$, the time used for observing in the next cycle.

Figure 3a shows interim results from the inference stage of the next cycle after making a single simulated observation at the optimal time. The period uncertainty has decreased by more than a factor of two, and the product of the posterior standard deviations of 

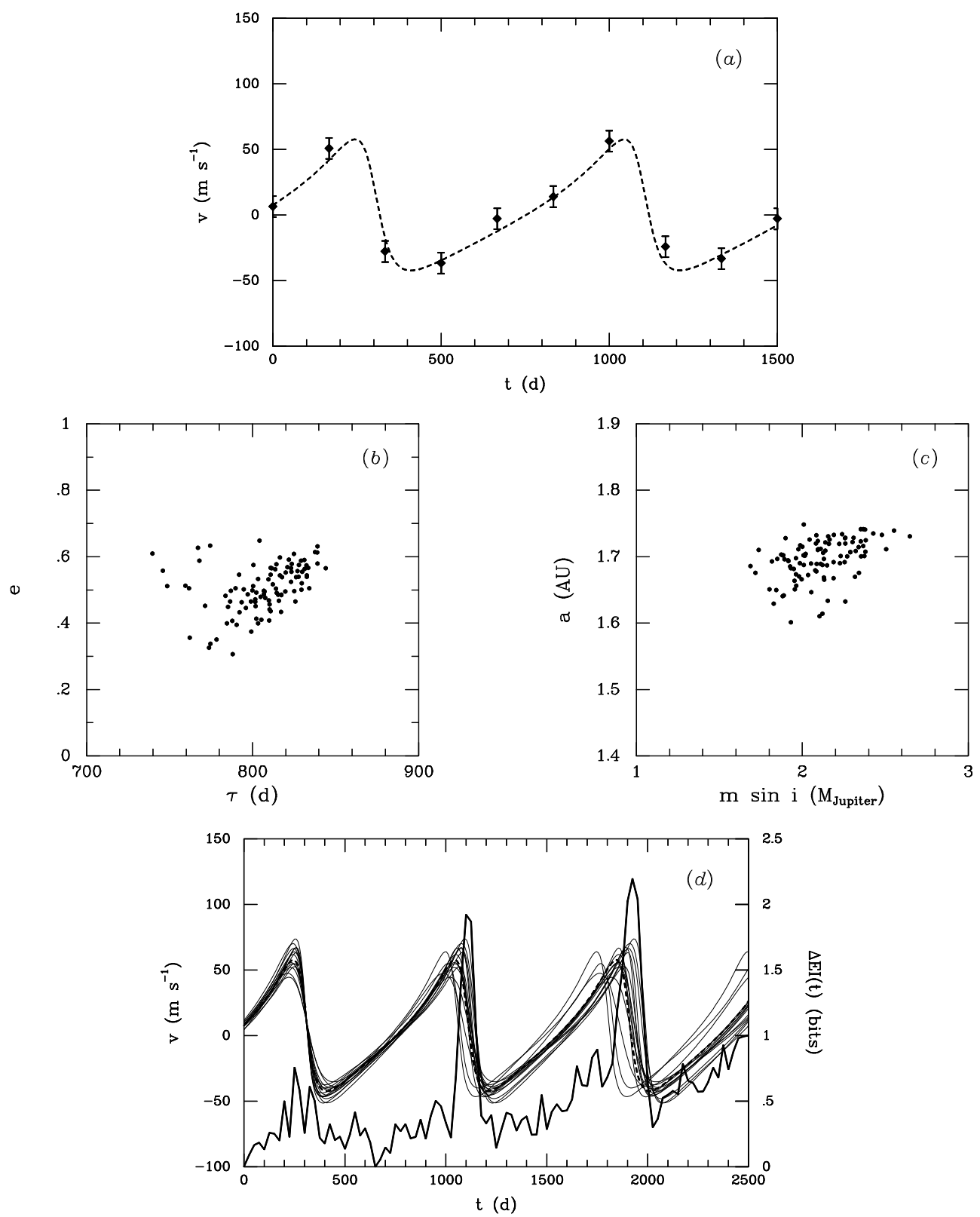

FIGURE 2. One cycle of the exploration process characterizing the orbit of an extrasolar planet with simulated radial velocity observations. (a) Observation stage, showing 10 simulated observations and true velocity curve (dashed). (b,c) Inference stage, showing samples from the posterior distribution for two velocity curve parameters (b) and two derived orbital parameters (c). (d) Design stage, showing predicted velocity curves (thin solid curves), true velocity curve (dashed curve), and the expected information gain for a sample at each time (thick solid curve, right axis).

all three parameters (the "posterior volume") has decreased by a factor $\approx 5.8$; this was accomplished by incorporating the information from a single well-chosen datum. Figures $3 b$ and $3 c$ show similar results from the next two cycles. The posterior volume 

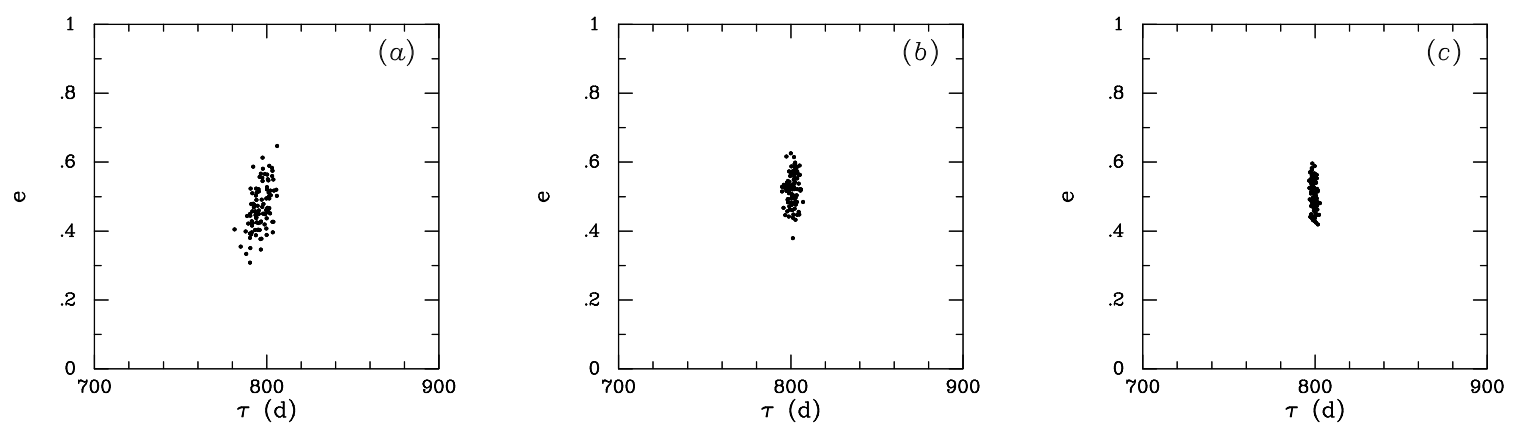

FIGURE 3. Inference stage results from three observation-inference-design cycles subsequent to that in Fig. 2, displaying rapid improvement of inferences.

continues to decrease much more rapidly than one would expect from the randomsampling " $\sqrt{N}$ rule" (by factors of $\approx 3.9$ and 1.8 ). Also, the correlation in the posterior is greatly reduced and is negligible in the final cycle.

\section{EXAMPLE: FINDING A HIDDEN OBJECT}

Figure 4 provides a further example motivated by the problem of detecting buried landmines using a mix of technologies-inexpensive but noisy ferromagnetic scans, and more costly but more sensitive acoustic scans using laser doppler vibrometry (see [21] for a discussion of some of this technology in a Bayesian setting). Figure 4a shows a hidden 1-d Gaussian-shaped "object" (dashed curve; peak at $x_{0}=5.2$, amplitude $A=7$, FWHM $=0.6)$ barely detected in an initial scan with 11 crude $(\sigma=1)$ observations spaced well over a full-width apart. Figure $4 \mathrm{~b}$ shows samples from the marginal posterior density for $A$ and $x_{0}$ from the first inference stage, displaying very substantial uncertainty. BAE proceeds, designing for subsequent more sensitive observations with reduced noise level $(\sigma=1 / 3)$. The design stage produces the entropy curve shown in Figure 4c (bold curve, right axis), and specifies observing near the best guess for the peak.

Figure 5 shows results from the inference and design stages of three subsequent BAE cycles. Incorporating data from a single observation taken as specified by Cycle 1 produces the more concentrated but complicated Cycle 2 inference of Figure 5a. Its "U" shape reflects the fact that the observations constrain the amplitude of the Gaussian at a particular point, but not the actual location of the Gaussian. Figure 5b shows the Cycle 2 design stage results, specifying an observation to the left of the estimated peak. Observing here produces the Cycle 3 inference in Figure 5c. Now the design stage directs attention to the other side of the object, as indicated by the entropy curve in Figure $5 \mathrm{~d}$. The subsequent Cycle 4 inference is shown in Figure 5e and is impressively accurate and uncorrelated. The posterior volume decreases by factors of $\approx 8.2,6.6$, and 5.6 between cycles, far more dramatically than expected from random sampling (even adjusting for the fact that only two of the original samples lie in the signal region). If for the last step one samples just a few tenths of a unit from the optimal point, the nonoptimal Cycle 4 inference in Figure 5f results; strong correlations remain, and the posterior volume is 

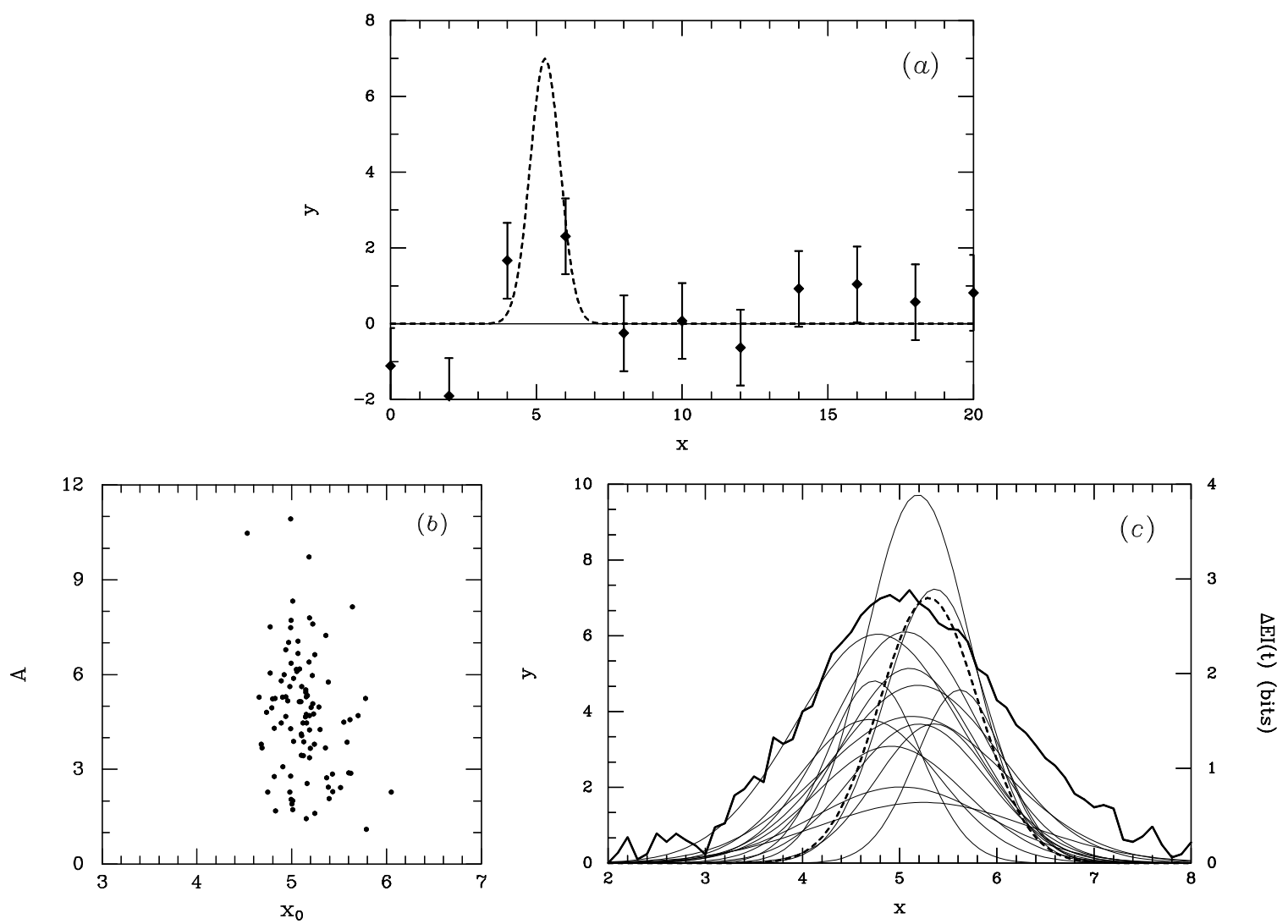

FIGURE 4. Results from the first observation-inference-design cycle for a simulated experiment characterizing a hidden 1-d Gaussian object with noisy observations.

$40 \%$ larger than what was obtained using an optimal observation.

\section{OPEN ISSUES}

The examples illustrate the BAE methodology and demonstrate its potential to very significantly improve observational efficiency in problems where one can adjust the sampling strategy on-the-fly to make inferences about well-specified nonlinear models. But several issues need to be addressed to make BAE useful in realistically complicated settings. The field of experimental design has a wide and diverse literature spread across several disciplines, and some of these topics are being addressed in current research under such titles as sequential design, active data selection, and active, adaptive, or incremental learning.

Evolving goals for inference. In both examples the goal was parameter estimation, the model being given. In reality, the goals of inference may not be so clear-cut. In exoplanet surveys, observers will often not be sure a system has a planetary companion at the start of an exploration, so the goal will initially be detection of a planet. Or if a system is targeted because it is known to have a planet, the goals may include detection of possible 

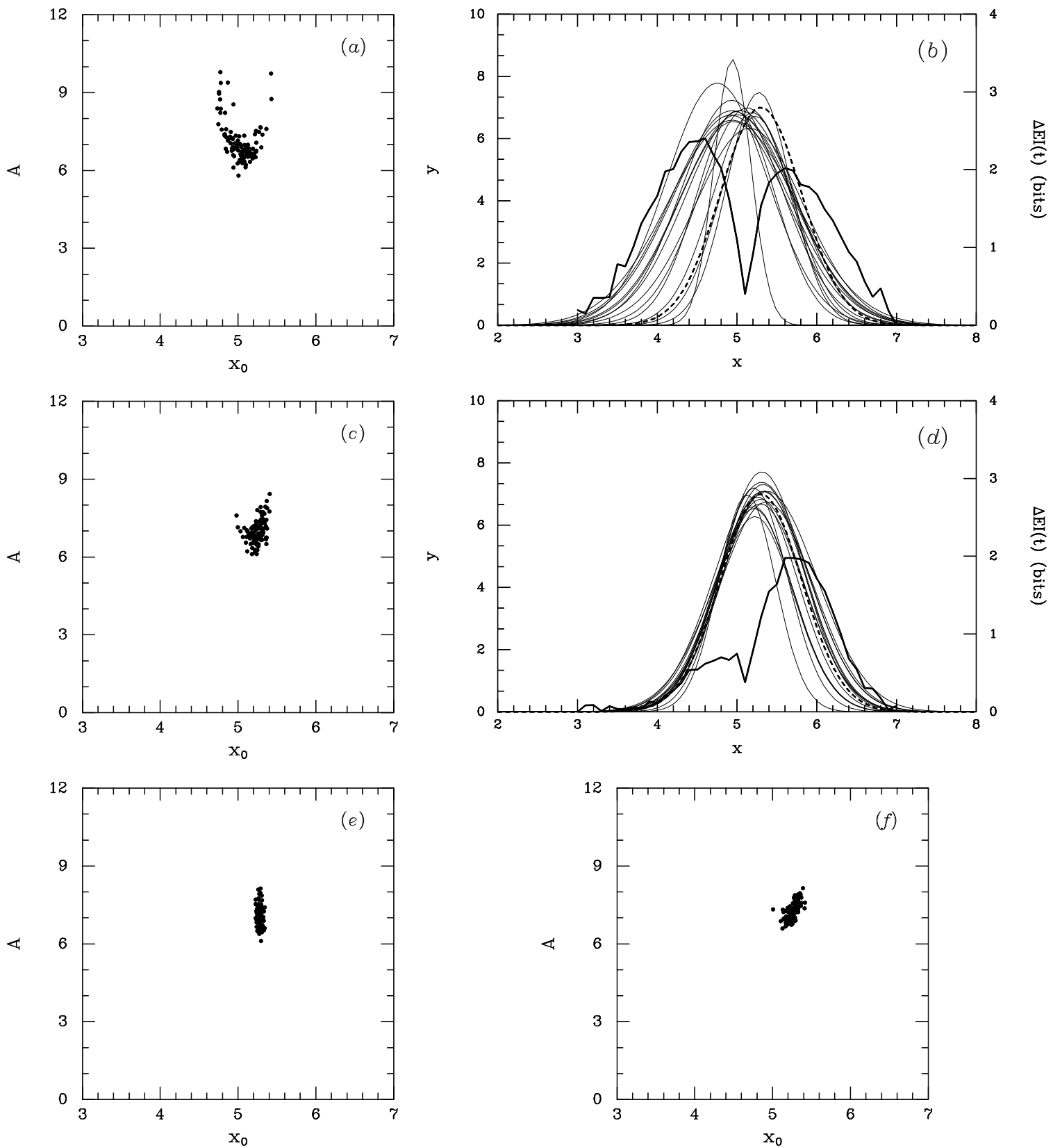

FIGURE 5. Results from the inference and design stages of three observation-inference-design cycles subsequent to the cycle shown in Fig. 4. Panel (f) shows inference stage results in the final cycle if a nonoptimal observation is used instead of the one specified in the previous design stage.

additional planets. At some point, the goal may shift from detection to estimation. In landmine detection, the goals will similarly shift from target detection to target classification (to distinguish rocks, debris, antipersonnel mines, and antitank mines), with parameter estimation largely subsidiary to these goals. How do design criteria for detection compare to those for estimation? When and how should the adaptive 
methodology shift its goal from detection to estimation? The work of Toman [22] on Bayesian design for multiple hypothesis testing provides a starting point for addressing these questions.

Generalizing the utility function. Our utility function was simply the information provided by new data. In some settings, one may wish to incorporate other elements in the utility function, such as the cost of observing as a function of time or sample size. How can a scientist map such costs to an information scale so that information and other costs or benefits can be combined into a single utility function?

Computational algorithms. We used a simple rejection method for generating posterior samples in our example. While attractively simple, such an approach is not useful for problems with more than five or six parameters (even fairly sophisticated envelope functions will waste too many samples). The obvious tool for addressing this is MCMC, but the Markov chain must ultimately sample over both the parameter space and the sample space (of future observations). Are there MCMC algorithms uniquely suited to adaptive exploration? Müller and Parmigiani and their colleagues [8, 9, 10, 11] have developed a variety of Monte Carlo approaches to Bayesian design in various settings that should be helpful in this regard, though, as here, they have so far treated fairly simple cases. Also, since adaptive exploration offers the hope of quickly reducing uncertainties, at some point it may make sense to linearize about the best-fit model and use analytic methods. Criteria need to be developed to identify when this is useful.

Design for the "setup" cycle. In our examples, the observing strategy for the first cycle was chosen somewhat arbitrarily. Ideally, it would be chosen using design principles and prior information. This raises many practical and theoretical questions. What should the size of a "setup" sample be? Should adaptive exploration start after a single sample, or are there benefits (perhaps associated with computational complexity) for starting with larger samples? Can the algorithms used for analysis when several samples are available also be used for designing the setup strategy, or are different algorithms required if prior information is very vague? Clearly, there is overlap between these issues and those already raised. This kind of design issue has been addressed informally for planning observations for the Hubble Space Telescope Cepheid key project [23]. Can a more formal approach improve on such a priori designs?

When is it worth implementing? Finally, though BAE provided impressive gains in the examples, such dramatic levels of improvement cannot be expected generally. Criteria must be identified to help determine when BAE or other formal design approaches may be useful. Measures of model nonlinearity and posterior nongaussianity may prove useful here. Also, it seems likely that the success of the approach depends critically on accurate model specification. Studies of robustness are needed to ascertain how performance might degrade with model inaccuracies, and whether the method can mislead investigators by avoiding parts of the sample space where one should search for departures from the model predictions (e.g., regions of the sample space where a model's predictions vary only very weakly with the parameters). Finally, is the approach ever valuable in settings with highly flexible models (e.g., semiparametric and nonparametric models)?

We hope this brief introduction will encourage scientists, engineers and statisticians to explore these issues together in a variety of contexts. 


\section{ACKNOWLEDGMENTS}

I thank David Chernoff for spurring my interest in adaptive design for SIM, for valuable discussions of the ideas presented here, and for coming up with the term, "Bayesian adaptive exploration." I also thank Paul Goggins for suggesting the landmine example and for valuable discussions about this example and other aspects of BAE. This work was supported by NASA through grant NAG5-12082 and through the SIM EPIcS Key Project.

\section{REFERENCES}

1. Fedorov, V. V., Theory of Optimal Experiments, Academic, New York, 1972.

2. Chernoff, H., Sequential Analysis and Optimal Design, SIAM, Philadelphia, 1972.

3. Atkinson, A. C., and Fedorov, V. V., "Optimum Design of Experiments," in Encyclopedia of statistical sciences, edited by S. Kotz, C. B. Read, and D. L. Banks, Wiley, New York, 1997, pp. 107-114.

4. Chaloner, K., and Verdinelli, I., Stat. Sci., 10, 273-304 (1995).

5. Toman, B., "Bayesian Experimental Design," in Encyclopedia of statistical sciences, Update Vol. 3, edited by S. Kotz and N. L. Johnson, Wiley, New York, 1999, pp. 35-39.

6. Sebastiani, P., and Wynn, H. P., "Bayesian experimental design and Shannon information," in 1997 Proceedings of the Section on Bayesian Statistical Science, American Statistical Association, 1997, pp. 176-181.

7. Sebastiani, P., and Wynn, H. P., J. Roy. Stat. Soc. B, 62, 145-157 (2000).

8. Muller, P., and Parmigiani, G., "Numerical evaluation of information theoretic measures," in Bayesian Statistics and Econometrics: Essays in Honor of A. Zellner, edited by D. A. Berry, K. M. Chaloner, and J. F. Geweke, Wiley, New York, 1995, pp. 397-406.

9. Clyde, M., Muller, P., and Parmigiani, G., "Exploring expected utility surfaces by markov chains," Tech. Rep. 95-39, ISDS, Duke University (1995).

10. Muller, P., and Parmigiani, G., J. Am. Stat. Assoc., 90, 1322-1330 (1996).

11. Muller, P., "Simulation based optimal design," in Bayesian Statistics 6, edited by J. O. Berger, J. M. Bernardo, A. P. Dawid, and A. F. M. Smith, 1999.

12. Loredo, T. J., and Chernoff, D. F., "Bayesian adaptive exploration," in Statistical Challenges in Astronomy, 2003, pp. 57-+.

13. Berger, J. O., Statistical Decision Theory and Bayesian Analysis, Springer-Verlag, New York, 1985.

14. Sivia, D., Data Analysis: A Bayesian Tutorial, Clarendon Press, Oxford, 1996.

15. Jaynes, E. T., Probability Theory: The Logic of Science, Cambridge University Press, Cambridge, 2003.

16. Lindley, D. V., Ann. Stat., 27, 986-1005 (1956).

17. Lindley, D. V., Bayesian statistics-a review, SIAM, Philadelphia, 1972.

18. MacKay, D., Neural Computation, 4, 590-604 (1992).

19. Loredo, T. J., "Computational Technology for Bayesian Inference," in ASP Conf. Ser. 172: Astronomical Data Analysis Software and Systems VIII, edited by D. M. Mehringer, R. L. Plante, and D. A. Roberts, Astronomical Society of the Pacific, San Francisco, 1999, pp. 297-306.

20. Sebastiani, P., and Settimi, R., J. Stat. Plan. Inf., 74, 177-192 (1998).

21. Xiang, N., and Goggans, P. M., J. Acoust. Soc. Am., 113, in press (2003).

22. Toman, B., J. Am. Stat. Assoc., 91, 185-190 (1996).

23. Freedman, W. L., Hughes, S. M., Madore, B. F., Mould, J. R., Lee, M. G., Stetson, P., Kennicutt, R. C., Turner, A., Ferrarese, L., Ford, H., Graham, J. A., Hill, R., Hoessel, J. G., Huchra, J., and Illingworth, G. D., Ap. J., 427, 628-655 (1994). 